\title{
Infranilmanifolds which admit complex contact structures
}

\author{
Yoshinobu Kamishima ${ }^{1}$
}

Received: 31 March 2015 / Accepted: 3 July 2015 / Published online: 25 July 2015

(C) Springer International Publishing AG 2015

\begin{abstract}
We study the complex nilpotent Lie group $\mathcal{L}$ which admits a complex contact structure and whose real subgroup admits a (real) contact structure. The group $\mathcal{L}$ is called the Iwasawa complex nilpotent Lie group. The homogeneous model of complex contact similarity geometry is introduced as $(\mathcal{L}, \operatorname{Sim}(\mathcal{L}))$ where $\operatorname{Sim}(\mathcal{L})$ is a transitive group of complex contact similarity transformations of $\mathcal{L}$. A smooth manifold locally modelled on this geometry is said to be a complex contact similarity manifold. We show that a compact complex contact similarity manifold can be determined under the conditions of developing pair.
\end{abstract}

Keywords Complex contact structure - Infranil-manifold · Iwasawa complex Lie group · Complex Boothby-Wang fibration · Complex affine group ·

Holonomy group $\cdot$ Developing map $\cdot$ Similarity geometry

Mathematics Subject Classification $\quad 53 \mathrm{D} 10 \cdot 57 \mathrm{~S} 25 \cdot 32 \mathrm{Q} 55$

\section{Introduction}

Let $\left(\operatorname{PSp}(n+1,1), \mathbb{H}_{\mathbb{H}}^{n+1}\right)$ be the homogeneous model of quaternionic hyperbolic geometry, where $\mathbb{H}$ is the quaternion number field. The hyperbolic action of $\operatorname{PSp}(n+1,1)$ extends to the quaternionic projective action on the boundary sphere $\partial \mathbb{H}_{\mathbb{H}}^{n+1}=S^{4 n+3}$ for which $\partial \mathbb{H}_{\mathbb{H}}^{n+1}=\operatorname{PSp}(n+1,1) / \operatorname{PSp}(n+1,1)_{\infty}$. Here $\operatorname{PSp}(n+1,1)_{\infty}$ is the stabilizer of the point at infinity $\infty \in \partial \mathbb{H}_{\mathbb{H}}^{n+1}$. The quaternionic

\footnotetext{
$\bigotimes$ Yoshinobu Kamishima

kami@josai.ac.jp

1 Department of Mathematics, Josai University, Keyaki-dai 1-1, Sakado, Saitama 350-0295, Japan
} 
$C R$-structure identifies the complement $S^{4 n+3}-\{\infty\}$ with the quaternionic Heisenberg nilpotent Lie group $\mathcal{M}$. The group $\mathcal{M}$ has the three dimensional center $\mathbb{R}^{3}=\operatorname{Im} \mathbb{H}$ which gives a central group extension $1 \rightarrow \mathbb{R}^{3} \rightarrow \mathcal{M} \longrightarrow \mathbb{H}^{n} \rightarrow 1$, where $\mathbb{H}^{n}$ is the quaternionic vector space. Taking a 1-dimensional central group $\mathbb{R}(=\mathbb{R} i)$ from $\mathbb{R}^{3}$, we obtain a quotient Lie group

$$
\mathcal{L}=\mathcal{M} / \mathbb{R}
$$

We call $\mathcal{L}$ the Iwasawa group. It is shown that $\mathcal{L}$ is a complex nilpotent Lie group (see Sect. 2). Recall that a complex contact structure on a complex $(2 n+1)$-dimensional complex manifold $M$ is a collection of local forms $\left\{U_{\alpha}, \omega_{\alpha}\right\}_{\alpha \in \Lambda}$ satisfying that $\bigcup_{\alpha \in \Lambda} U_{\alpha}=M$, each $\omega_{\alpha}$ is a holomorphic 1-form defined on $U_{\alpha}$ such that $\omega_{\alpha} \wedge\left(d \omega_{\alpha}\right)^{n} \neq 0$ on $U_{\alpha}$ and if $U_{\alpha} \cap U_{\beta} \neq \emptyset$, then there exists a nonzero holomorphic function $f_{\alpha \beta}$ on $U_{\alpha} \cap U_{\beta}$ such that $f_{\alpha \beta} \cdot \omega_{\alpha}=\omega_{\beta}$.

We prove that $\mathcal{L}$ admits an invariant complex contact structure canonically by constructing a global holomorphic 1-form $\eta$ endowed with the group of complex contact transformations $\mathrm{A}(\mathcal{L})$ in Sects. 3 and 4 (see [10, Section 4]). In this paper we take a specific subgroup $\operatorname{Sim}(\mathcal{L})$ called a complex contact similarity group from $\mathrm{A}(\mathcal{L})$. In general, the pair $(\operatorname{Sim}(\mathcal{L}), \mathcal{L})$ is said to be the homogeneous model of complex contact similarity geometry (cf. Sect. 6). We study compact complex contact manifolds modelled on $(\operatorname{Sim}(\mathcal{L}), \mathcal{L})$. As usual, given a uniformization with respect to $(\operatorname{Sim}(\mathcal{L}), \mathcal{L})$ on a manifold $M$, there exist a developing immersion dev : $\widetilde{M} \rightarrow \mathcal{L}$ and a holonomy homomorphism $\rho: \pi_{1}(M) \rightarrow \operatorname{Sim}(\mathcal{L})$ respectively. Here $\pi_{1}(M)$ is the fundamental group of $M$. The image $\rho\left(\pi_{1}(M)\right)$ is said to be the holonomy group for $M$. We prove the following uniformization.

Theorem A Let $M$ be a $(4 n+2)$-dimensional compact complex contact similarity manifold. Suppose that either of the following holds.

(a) The holonomy group is virtually nilpotent.

(b) The holonomy group is discrete.

Then $M$ is biholomorphic to either a complex contact Iwasawa infranilmanifold $\mathcal{L} / \Gamma$ or a complex contact infra-Hopfmanifold $\mathcal{L}-\{0\} / \Gamma$. Here $\Gamma$ is the holonomy group isomorphic to either a discrete cocompact virtually nilpotent subgroup in $\mathcal{L} \rtimes\left(\operatorname{Sp}(n) \cdot S^{1}\right)$ or the product $\mathbb{Z}_{p} \times \mathbb{Z}^{+}$in $\mathrm{Sp}(n) \cdot S^{1} \times \mathbb{R}^{+}$respectively.

Theorem A may be true without condition (a) or (b). The supporting examples were obtained by Fried (Euclidean similarity manifold) [5] and Miner (Heisenberg similarity manifold) [17]. However, there is an embedding of $\operatorname{Sim}(\mathcal{L})$ into the complex affine group Aff $\left(\mathbb{C}^{2 n+1}\right)$ (cf.(5)). By Proposition 5.3, $M$ is also a complex affinely flat manifold. Thus, the determination of $M$ is also related to the Auslander-Milnor conjecture (cf. [6]).

We discuss the deformation of the complex contact Iwasawa infranilmanifold $\mathcal{L} / \Gamma$ as a holomorphic Seifert fibering (cf. [15]) in Sect. 6.1. As in Theorem A, the developing pair $(\rho, \mathrm{dev})$ is a geometric invariant for complex contact similarity manifolds. In Sect. 7, we study a compactification which shows that $\mathcal{L} \cup\{\infty\}$ is homeomorphic to the $(4 n+2)$-sphere. By using developing maps we characterize $M$ (see Theorem 7.2). 
Theorem B If the developing map of a compact $(4 n+2)$-dimensional complex contact similarity manifold $M$ is not surjective onto $\mathcal{L}$, then $\operatorname{dev}$ is a biholomorphim of $\widetilde{M}$ onto $\mathcal{L}-\{0\}$.

\section{Iwasawa Lie group}

Let $J_{n}$ be the complex structure $\overbrace{J \oplus \cdots \oplus J}^{n}$ on $\mathbb{C}^{2 n}$, where $J=\left(\begin{array}{cc}0 & 1 \\ -1 & 0\end{array}\right)$. The quaternionic vector space $\mathbb{H}^{n}$ is identified with $\mathbb{C}^{2 n}$ by the isomorphism

$$
\left(z_{1}+z_{2} j, \ldots, z_{2 n-1}+z_{2 n} j\right) \mapsto\left(\bar{z}_{1}, z_{2}, \ldots, \bar{z}_{2 n-1}, z_{2 n}\right)
$$

Our group $\mathcal{L}$ is defined to be the product $\mathbb{C}^{2 n+1}=\mathbb{C} \times \mathbb{H}^{n}$ with the group law

$$
\begin{aligned}
(x, z) \cdot(y, w) & =\left(x+y+\sum_{i=1}^{n} z_{2 i-1} w_{2 i}-z_{2 i} w_{2 i-1}, z+w\right) \\
& =\left(x+y+{ }^{t} z \mathrm{~J}_{n} w, z+w\right), \quad n \geq 1,
\end{aligned}
$$

where $x, y \in \mathbb{C}, z_{k} \in \mathbb{C}, w_{k} \in \mathbb{C}, k=1, \ldots, 2 n,{ }^{t} z=\left(z_{1}, \ldots, z_{2 n}\right)=\left(\bar{z}_{1}+z_{2} j\right.$, $\left.\ldots, \bar{z}_{2 n-1}+z_{2 n} j\right) \in \mathbb{H}^{n}$ and ${ }^{t} w=\left(w_{1}, \ldots, w_{2 n}\right)=\left(\bar{w}_{1}+w_{2} j, \ldots, \bar{w}_{2 n-1}+\right.$ $\left.w_{2 n} j\right) \in \mathbb{H}^{n}$.

It is easy to see that $[(x, z),(y, w)]=\left(2 \sum_{i=1}^{n} z_{2 i-1} w_{2 i}-z_{2 i} w_{2 i-1}, 0\right)$ so $[\mathcal{L}, \mathcal{L}]=(\mathbb{C},(0, \ldots, 0))=\mathbb{C}$ which is the center of $\mathcal{L}=\mathcal{L}_{n}$. It has a central group extension $1 \rightarrow \mathbb{C} \rightarrow \mathcal{L}_{n} \longrightarrow \mathbb{C}^{2 n} \rightarrow 1$.

Moreover, let $L_{h}: \mathcal{L} \rightarrow \mathcal{L}$ be a left translation for $h=(x, z) \in \mathcal{L}$ defined by $L_{h}(y, w)=h \cdot(y, w)=(x, z) \cdot(y, w)$. Consider the differentiable map $L_{h *}: T_{(y, w)} \mathcal{L} \rightarrow T_{h \cdot(y, w)} \mathcal{L}$. For $(X, Y) \in T_{(y, w)} \mathcal{L}=T_{y} \mathbb{C} \times T_{w} \mathbb{C}^{2 n}$, it follows

$$
L_{h *}(X, Y)=\lim _{t \rightarrow 0} \frac{1}{t}\left(L_{h}(y+t X, w+t Y)-L_{h}(y, w)\right)=\left(X+{ }^{t} z \mathrm{~J}_{n} Y, Y\right) .
$$

Let $J_{0}$ be the standard complex structure on $\mathcal{L}=\mathbb{C}^{2 n+1}$. As $J_{0}(X, Y)=(i X, i Y)$, the above equation implies $L_{h *} \circ J_{0}=J_{0} \circ L_{h *}$ obviously, i.e. every left translation is a holomorphic transformation on $\mathcal{L}$. Similar to this argument, it is easily checked that the correspondences $((x, z),(y, w)) \mapsto(x, z) \cdot(y, w)=\left(x+y+{ }^{t} z \mathrm{~J}_{n} w, z+w\right)$ and $(x, z) \mapsto(x, z)^{-1}=(-x,-z)$ are also holomorphic. Thus $\mathcal{L}$ is a complex Lie group.

Definition 2.1 A complex $(2 n+1)$-dimensional complex nilpotent Lie group $\mathcal{L}_{n}$ is said to be the Iwasawa Lie group.

Remark 2.2 It is easy to check that $\mathcal{L}_{1}$ is isomorphic to the Iwasawa group consisting of $3 \times 3$-upper triangular unipotent complex matrices by the correspondence

$$
\left(x,\left(z_{1}, z_{2}\right)\right)=\left(\begin{array}{ccc}
1 & z_{1} & z_{3} \\
0 & 1 & z_{2} \\
0 & 0 & 1
\end{array}\right), \quad z_{3}=\frac{z_{1} z_{2}+x}{2} .
$$




\section{Existence of complex contact structure}

\subsection{Holomorphic 1-form}

Let $\left(z_{0}, z_{1}, \ldots, z_{2 n}\right)$ be the standard coordinates of $\mathcal{L}$. We define a complex 1 -form $\eta$ as follows:

$$
\eta=d z_{0}-\sum_{i=1}^{n} z_{2 i-1} \cdot d z_{2 i}-z_{2 i} \cdot d z_{2 i-1}=d z_{0}-{ }^{t} z \mathbf{J}_{n} d z
$$

where ${ }^{t} z=\left(z_{1}, \ldots, z_{2 n}\right),{ }^{t} d z=\left(d z_{1}, \ldots, d z_{2 n}\right)$. Since $\eta \wedge(d \eta)^{n}$ is a non-vanishing form $n(-1)^{n}(-2)^{n} d z_{0} \wedge \cdots \wedge d z_{2 n}$ on $\mathcal{L}, \eta$ is a complex contact structure (globally defined) on $\mathcal{L}$ (see [12] for the definition).

\subsection{Real points of $\mathcal{L}$}

The group $\mathcal{L}$ contains the real point set $\mathcal{N}=\mathbb{R} \times \mathbb{C}^{n}$ which has an induced group structure

$$
(a, x) \cdot(b, y)=\left(a+b+\sum_{i=1}^{n} x_{2 i-1} y_{2 i}-x_{2 i} y_{2 i-1}, x+y\right),
$$

where ${ }^{t} x=\left(x_{1}+x_{2} j, \ldots, x_{2 n-1}+x_{2 n} j\right) \in \mathbb{C}^{n}, x_{k} \in \mathbb{R}$, and ${ }^{t} y=\left(y_{1}+y_{2} j, \ldots\right.$, $\left.y_{2 n-1}+y_{2 n} j\right) \in \mathbb{C}^{n}, y_{k} \in \mathbb{R}$ (here we use $j$ as a complex structure instead of $i$ ). Obviously, $\mathcal{N}$ is also a 2 -step nilpotent Lie subgroup of $\mathcal{L}$ such that $T \mathcal{N} \oplus i T \mathcal{N}=T \mathcal{L}$. By the definition, $\mathcal{N}$ is the Heisenberg nilpotent Lie group. The complex contact structure $\eta$ restricts it to a (real) 1 -form

$$
\omega=d x_{0}-\sum_{i=1}^{n} x_{2 i-1} \cdot d x_{2 i}-x_{2 i} \cdot d x_{2 i-1}
$$

which satisfies $\omega \wedge(d \omega)^{n} \equiv d x_{0} \wedge d x_{1} \wedge \cdots \wedge d x_{2 n} \neq 0$ (up to constant). Moreover, the pair $(\operatorname{ker} \eta, J)$ is a $C R$-structure on $\mathcal{N}$.

Remark 3.1 Let $1 \rightarrow \mathbb{R} \rightarrow \mathcal{M} \stackrel{v}{\longrightarrow} \mathcal{L} \rightarrow 1$ be the central group extension as before. The canonical quaternionic $C R$-structure on $\mathcal{M}$ defines a Carnot-Carathéodory structure $(B,\{I, J, K\})$. Then one of the almost complex structures $I$ is integrable on $D=T \mathbb{C} \oplus B$ (cf. [1]). In [10] we have shown that the holomorphic $2 n$-dimensional bundle $D^{1,0}$ maps isomorphically onto a complex contact subbundle $v_{*}(D)^{1,0}$ on $\mathcal{L}$, i.e. $\left[v_{*}(D)^{1,0}, v_{*}(D)^{1,0}\right] \equiv \mathbb{C} \bmod v_{*}(D)^{1,0}$. Note that the above extension is a principal bundle but not a 3-Sasakian space. So this complex contact structure on $\mathcal{L}$ does not come from any Sasakian 3 -structure or any twistor space (cf. [7,18]). As a consequence, $\eta$ turns out to be a representative (global) holomorphic 1-form on $\mathcal{L}$. 


\section{The group of complex contact transformations}

Let hol $(\mathcal{L})$ be the group of biholomorphic transformations of $\left(\mathcal{L}, J_{0}\right)$. The group of complex contact transformations on $(\mathcal{L}, \eta)$ is denoted by

$$
\operatorname{hol}(\mathcal{L}, \eta)=\left\{f \in \operatorname{hol}(\mathcal{L}): f^{*} \eta=\tau \cdot \eta\right\}
$$

for some holomorphic function $\tau$ on $\mathcal{L}$. Let $\operatorname{Sp}(n ; \mathbb{C})=\left\{A \in M(2 n, \mathbb{C}):{ }^{t} A \mathrm{~J}_{n} A=\right.$ $\left.\mathrm{J}_{n}\right\}$ be the complex symplectic group. Put

$$
\mathrm{A}(\mathcal{L})=\mathcal{L} \rtimes\left(\operatorname{Sp}(n ; \mathbb{C}) \cdot \mathbb{C}^{*}\right)
$$

which is the semidirect product; let $\lambda \cdot A, \mu \cdot B \in \operatorname{Sp}(n ; \mathbb{C}) \cdot \mathbb{C}^{*}$ for $A, B \in \operatorname{Sp}(n ; \mathbb{C})$, $\lambda, \mu \in \mathbb{C}^{*}$ and $(a, w),(b, z) \in \mathcal{L}$. This forms a group by defining

$$
\begin{aligned}
((a, w), \lambda \cdot A) \cdot & ((b, z), \mu \cdot B) \\
= & ((a, w), I) \cdot\left(\left(\lambda^{2} b, \lambda A z\right), \lambda \cdot A \cdot \mu \cdot B\right) \\
& =\left(\left(a+\lambda^{2} b+{ }^{t} w J_{n}(\lambda A z), w+\lambda A z\right), \lambda \mu \cdot A B\right) .
\end{aligned}
$$

The group $\mathrm{A}(\mathcal{L})$ acts on $\mathcal{L}$ as follows. For $((a, w), \lambda \cdot A) \in \mathrm{A}(\mathcal{L}),\left(z_{0}, z\right) \in \mathcal{L}$, define

$$
\begin{aligned}
((a, w), \lambda \cdot A) \cdot\left(z_{0}, z\right) & =(a, w) \cdot\left(\lambda^{2} z_{0}, \lambda A z\right) \\
& =\left(a+\lambda^{2} z_{0}+{ }^{t} w \mathrm{~J}_{n}(\lambda A z), w+\lambda A z\right) .
\end{aligned}
$$

If $h=((b, w), \mu \cdot B) \in \mathrm{A}(\mathcal{L})$ with $\mu \in \mathbb{C}^{*}$, then it is easy to see that

$$
h^{*} \eta=\mu^{2} \cdot \eta \text {. }
$$

We have to check that each element of $\mathrm{A}(\mathcal{L})$ is holomorphic. Consider the complex affine group Aff $\left(\mathbb{C}^{2 n+1}\right)=\mathbb{C}^{2 n+1} \rtimes \mathrm{GL}(2 n+1, \mathbb{C})$. We assign to each $((a, w), \lambda \cdot A) \in \mathrm{A}(\mathcal{L})$ an element

$$
\left(\left[\begin{array}{c}
a \\
w
\end{array}\right],\left(\begin{array}{c|c}
\lambda^{2} & \lambda^{t} w \mathrm{~J}_{n} A \\
\hline 0 & \lambda A
\end{array}\right)\right) \in \operatorname{Aff}\left(\mathbb{C}^{2 n+1}\right) .
$$

It is easy to see that this correspondence is an injective homomorphism. Since $\mathcal{L}=$ $\mathbb{C}^{2 n+1}$, by this assignment the action $(2)$ of $((a, w), \lambda \cdot A)$ on $\mathcal{L}$ coincides with the complex affine transformation (4) of $\mathbb{C}^{2 n+1}$. In particular, we obtain

$$
\mathrm{A}(\mathcal{L}) \leq \operatorname{Aff}\left(\mathbb{C}^{2 n+1}\right)(\leq \operatorname{hol}(\mathcal{L}))
$$

Combined with $(3), \mathrm{A}(\mathcal{L})$ preserves the complex contact structure on $(\mathcal{L}, \eta)($ see $(1))$.

Remark 4.1 Since $\eta_{\mid \mathcal{N}}=\omega, \mathrm{A}(\mathcal{L})$ restricted to $\mathcal{N}$ is the group of $C R$-transformations $\operatorname{Aut}_{C R}(\mathcal{N})=\left\{f \in \operatorname{Diff}(\mathcal{N}): f^{*} \omega=\mu \cdot \omega\right\}$ for some smooth function $\mu$ on $\mathcal{N}$. 


\section{Uniformization over $(\mathrm{A}(\mathcal{L}), \mathcal{L})$}

\subsection{Complex contact structure and transformations}

Recall from [12] that a complex contact structure on a complex $(2 n+1)$-dimensional complex manifold $M$ is a collection of local forms $\left\{U_{\alpha}, \omega_{\alpha}\right\}_{\alpha \in \Lambda}$ satisfying that (a) $\bigcup_{\alpha \in \Lambda} U_{\alpha}=M$, (b) each $\omega_{\alpha}$ is a holomorphic 1-form defined on $U_{\alpha}$ such that $\omega_{\alpha} \wedge\left(d \omega_{\alpha}\right)^{n} \neq 0$ on $U_{\alpha}$ and (c) if $U_{\alpha} \cap U_{\beta} \neq \emptyset$, then there exists a nonzero holomorphic function $f_{\alpha \beta}$ on $U_{\alpha} \cap U_{\beta}$ such that $f_{\alpha \beta} \cdot \omega_{\alpha}=\omega_{\beta}$.

Let $h: M \rightarrow M$ be a biholomorphism. Suppose that $h\left(U_{\alpha}\right) \cap U_{\beta} \neq \varnothing$ for some $\alpha, \beta \in \Lambda$. If there exists a holomorphic function $f_{\alpha \beta}$ defined on a neighborhood of $U_{\alpha}$ such that $h^{*} \omega_{\beta}=f_{\alpha \beta} \cdot \omega_{\alpha}$, then we call $h$ a complex contact transformation of $M$. This is consistent with the definition of (1).

Denote by $\operatorname{Aut}_{\mathrm{cc}}(M)$ the group of complex contact transformations (when $M$ is compact, note that $\operatorname{Aut}_{\mathrm{cc}}(M)$ is a finite dimensional Lie group). Let $\widetilde{M}$ be the universal covering manifold of $M$ and $\pi$ its fundamental group $\pi_{1}(M)$. Naturally, by taking a lift of complex contact structure of $M, \widetilde{M}$ is a complex contact manifold for which $\pi \leq \operatorname{Aut}_{\mathrm{cc}}(\widetilde{M})$. If $N_{\mathrm{Aut}_{\mathrm{cc}}(\widetilde{M})}(\pi)$ is the normalizer of $\pi$, then there is an exact sequence

$$
1 \rightarrow \pi \rightarrow N_{\operatorname{Aut}_{\mathrm{cc}}(\widetilde{M})}(\pi) \rightarrow \operatorname{Aut}_{\mathrm{cc}}(M) \rightarrow 1
$$

\subsection{Uniformization and developing map}

Let $M$ be a smooth (not necessarily complex) manifold. Suppose that there exists a maximal collection of charts $\left\{\left(U_{\alpha}, \varphi_{\alpha}\right)\right\}_{\alpha \in \Lambda}$ whose coordinate changes belong to $\mathrm{A}(\mathcal{L})$. More precisely, $M=\bigcup_{\alpha \in \Lambda} U_{\alpha}, \varphi_{\alpha}: U_{\alpha} \rightarrow \mathcal{L}$ is a diffeomorphism onto its image. If $U_{\alpha} \cap U_{\beta} \neq \emptyset$, then there exists a unique element $g_{\alpha \beta} \in \mathrm{A}(\mathcal{L})$ such that $g_{\alpha \beta}=\varphi_{\beta} \cdot \varphi_{\alpha}^{-1}$ on $\varphi_{\alpha}\left(U_{\alpha} \cap U_{\beta}\right)$. We say that $M$ is locally modelled on $(\mathrm{A}(\mathcal{L}), \mathcal{L})$ (compare [14]). The equivalence class of such a maximal collection of charts is said to be a uniformization with respect to $(\mathrm{A}(\mathcal{L}), \mathcal{L})$.

Given a uniformization on a manifold $M$, the monodromy argument shows that there exist a developing immersion $\operatorname{dev}: \widetilde{M} \rightarrow \mathcal{L}$ and a holonomy homomorphism $\rho: \operatorname{Aut}_{\mathrm{cc}}(\widetilde{M}) \rightarrow \mathrm{A}(\mathcal{L})$ respectively. By the construction (cf. Proposition 5.1), there exists a complex structure $J$ on $M$ such that $\operatorname{dev}_{*} \circ J=J_{0} \circ \operatorname{dev}_{*}$ (we use the same notation $J$ on $\widetilde{M}$ ). So dev is a holomorphic immersion. Here is a sufficient condition for the existence on complex contact structure.

Proposition 5.1 If a $(4 n+2)$-dimensional smooth manifold $M$ is locally modelled on $(\mathrm{A}(\mathcal{L}), \mathcal{L})$, then $M$ admits a complex contact structure.

Proof First of all we define a complex structure on $M$. Let $J_{0}$ be the standard complex structure on $\mathcal{L}=\mathbb{C}^{2 n+1}$. Define a complex structure $J_{\alpha}$ on $U_{\alpha}$ by setting $\varphi_{\alpha *} J_{\alpha}=$ $J_{0} \varphi_{\alpha *}$ on $U_{\alpha}$ for each $\alpha \in \Lambda$. When $h \in \mathrm{A}(\mathcal{L})$, note that $h_{*} J_{0}=J_{0} h_{*}$ from (5). If $U_{\alpha} \cap U_{\beta} \neq \emptyset$, then there exists a unique element $g_{\alpha \beta} \in \mathrm{A}(\mathcal{L})$ such that $g_{\alpha \beta}=\varphi_{\beta} \cdot \varphi_{\alpha}^{-1}$. A calculation shows that $\varphi_{\beta *} J_{\alpha}=g_{\alpha \beta *} \varphi_{\alpha *} J_{\alpha}=J_{0} \varphi_{\beta *}$. Since $\varphi_{\beta *} J_{\beta}=J_{0} \varphi_{\beta *}$ by the 
definition, it follows $J_{\alpha}=J_{\beta}$ on $U_{\alpha} \cap U_{\beta}$. This defines a complex structure $J$ on $M$. In particular, each $\varphi_{\alpha}:\left(U_{\alpha}, J\right) \rightarrow\left(\mathcal{L}, J_{0}\right)$ is a holomorphic embedding.

Let $\eta$ be the holomorphic 1-form on $\mathcal{L}$ as before. Define a family of local holomorphic 1 -forms $\left\{\omega_{\alpha}, U_{\alpha}\right\}_{\alpha \in \Lambda}$ by

$$
\omega_{\alpha}=\varphi_{\alpha}^{*} \eta \quad \text { on } \quad U_{\alpha}
$$

From (3), $g_{\alpha \beta}^{*} \eta=\mu_{\alpha \beta}^{2} \cdot \eta$ for some $\mu_{\alpha \beta} \in \mathbb{C}^{*}$. It follows $\omega_{\beta}=\mu_{\alpha \beta}^{2} \cdot \omega_{\alpha}$. Thus the family $\left\{\omega_{\alpha}, U_{\alpha}\right\}_{\alpha \in \Lambda}$ gives rise to a complex contact structure on $(M, J)$.

Remark 5.2 When a subgroup $\Gamma \leq \mathrm{A}(\mathcal{L})$ acts properly discontinuously and freely on a domain $\Omega$ of $\mathcal{L}$ with compact quotient, we obtain a compact complex contact manifold $\Omega / \Gamma$ by this proposition. In this case, when we put $M=\Omega / \Gamma$, the developing map is the covering map $\widetilde{M}=\widetilde{\Omega} \rightarrow \Omega \subset \mathcal{L}$ and the holonomy homomorphism is a projection $p: \widetilde{\Gamma} \rightarrow \Gamma$, where $1 \rightarrow \pi_{1}(\Omega) \rightarrow \widetilde{\Gamma} \stackrel{p}{\rightarrow} \Gamma \rightarrow 1$ is an exact sequence. In general it is too difficult to find such a properly discontinuous cocompact subgroup $\Gamma$.

On the other hand, we have the embedding (5) (cf.(4)), this induces another uniformization simultaneously.

Proposition 5.3 A $(4 n+2)$-dimensional smooth manifold $M$ locally modelled on $(\mathrm{A}(\mathcal{L}), \mathcal{L})$ supports a complex affine flat structure.

When the developing map dev: $\widetilde{M} \rightarrow \mathcal{L}=\mathbb{C}^{2 n+1}$ is a diffeomorphism, $\mathbb{C}^{2 n+1} / \Gamma$ is said to be a compact complete complex affinely flat manifold. Concerning the Auslander-Milnor conjecture, we do not know whether the fundamental group $\Gamma \leq \mathrm{A}(\mathcal{L})$ is virtually polycyclic (compare [16]).

\section{Complex contact similarity geometry}

In view of Remark 5.2 and the Auslander-Milnor conjecture below Proposition 5.3, we may find a smaller subgroup of $\mathrm{A}(\mathcal{L})$ in order to get compact complex contact manifolds with nontrivial fundamental groups. First of all, $\operatorname{Sp}(n, \mathbb{C})$ contains a maximal compact symplectic subgroup $\operatorname{Sp}(n)=\left\{A \in \mathrm{U}(2 n):{ }^{t} A J_{n} A=J_{n}\right\}$ where $\mathrm{Sp}(n, \mathbb{C}) \cong \mathrm{Sp}(n) \times \mathbb{R}^{n(2 n+1)}$.

Definition 6.1 Put $\operatorname{Sim}(\mathcal{L})=\mathcal{L} \rtimes\left(\operatorname{Sp}(n) \cdot \mathbb{C}^{*}\right) \leq \mathrm{A}(\mathcal{L})$. The pair $(\operatorname{Sim}(\mathcal{L}), \mathcal{L})$ is called the homogeneous model of complex contact similarity geometry. A manifold locally modelled on this geometry is said to be a complex contact similarity manifold (cf. Proposition 5.1). The Euclidean subgroup $\mathrm{E}(\mathcal{L})$ of $\operatorname{Sim}(\mathcal{L})$ is defined to be $\mathcal{L} \rtimes\left(\operatorname{Sp}(n) \cdot S^{1}\right)$.

We give two typical examples of compact complex contact similarity manifolds.

(I) Infra-Hopf manifold. Choose $\lambda \in \mathbb{C}^{*}$ with $|\lambda| \neq 1$ and put $r=((0,0), \lambda) \in$ $\operatorname{Sim}(\mathcal{L})$. Denote by $\mathbb{Z}^{+}$an infinite cyclic group generated by $r$. Let $\mathcal{L}-\{0\}$ be the 
complement with the origin $0=(0,0)$ removed. Then it is easy to see that $\mathbb{Z}^{+}$acts freely and properly discontinuously on $\mathcal{L}-\{0\}$ by (2):

$$
r\left(z_{0}, z\right)=\left(\lambda^{2} z_{0}, \lambda z\right)
$$

The quotient $\mathcal{L}-\{0\} / \mathbb{Z}^{+}$is diffeomorphic to $S^{1} \times S^{4 n+1}$. By Proposition 5.1 (cf. Remark 5.2), $S^{1} \times S^{4 n+1}$ is a complex contact similarity manifold on which $\operatorname{Sp}(n) \cdot S^{1} \times S^{1}=\operatorname{Sp}(n) \cdot \mathbb{C}^{*} /\langle r\rangle$ acts properly as complex contact transformations. If $\mathbb{Z}_{p} \leq \operatorname{Sp}(n) \cdot S^{1} \times S^{1}$ is a cyclic subgroup of order $p>1$ acting freely on $S^{1} \times S^{4 n+1}$, then the quotient by the action $\mathbb{Z}_{p}$ is an infra-Hopf manifold.

(II) Infranilmanifold. Let $\Gamma$ be a torsionfree discrete cocompact subgroup of $\mathrm{E}(\mathcal{L})$. By the Auslander-Bieberbach theorem, $\Gamma \cap \mathcal{L}$ is discrete uniform in $\mathcal{L}$. Since $\mathbb{C}$ is the central subgroup of $\mathcal{L}, \Gamma \cap \mathbb{C}$ is a discrete uniform subgroup $\mathbb{Z}^{2}$ in $\mathbb{C}$. We have the following commutative diagram of exact sequences:

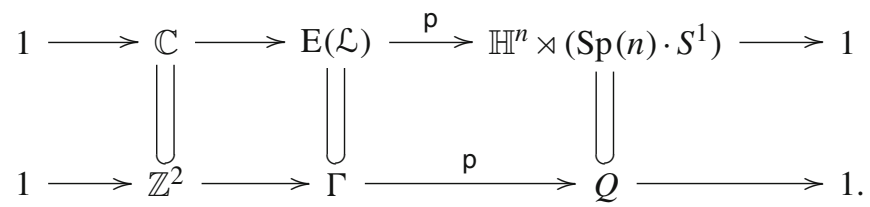

As a consequence, the quotient group $Q=\mathrm{p}(\Gamma)$ is a discrete uniform subgroup in $\mathbb{H}^{n} \rtimes\left(\operatorname{Sp}(n) \cdot S^{1}\right) \leq \mathrm{E}\left(\mathbb{H}^{n}\right)$. In particular, the quotient $\mathbb{H}^{n} / Q$ is a compact quaternionic Euclidean orbifold. Letting $T_{\mathbb{C}}^{1}=\mathbb{C} / \mathbb{Z}^{2}$, we obtain a compact $(4 n+2)$-dimensional complex contact Iwasawa infranilmanifold $\mathcal{L} / \Gamma$ which supports a holomorphic Seifert fibering $T_{\mathbb{C}}^{1} \rightarrow \mathcal{L} / \Gamma \longrightarrow \mathbb{H}^{n} / Q$ (compare [15]).

Remark 6.2 Since there exists a finite index normal free abelian subgroup $\mathbb{Z}^{4 n} \leq \mathbb{H}^{n}$ of $Q$ by the Bieberbach theorem, this induces a central group extension $1 \rightarrow \mathbb{Z}^{2} \rightarrow \Delta \rightarrow$ $\mathbb{Z}^{4 n} \rightarrow 1$ such that $\Delta \leq \mathcal{L}$. A complex contact nilmanifold $\mathcal{L} / \Delta$ admits a holomorphic principal $T_{\mathbb{C}}^{1}$-bundle over a complex torus $T_{\mathbb{C}}^{2 n}=\mathbb{H}^{n} / \mathbb{Z}^{4 n}$. This example is a special case of Foreman's $T^{2}$-connection bundle over $T_{\mathbb{C}}^{2 n}$ (cf. [3]). As a consequence, a compact complex contact Iwasawa infranilmanifold $\mathcal{L} / \Gamma$ is finitely covered by $\mathcal{L} / \Delta$.

\subsection{Deformation of Iwasawa infranilmanifold $\mathcal{L} / \Gamma$}

Let $1 \rightarrow \mathbb{Z}^{2} \rightarrow \Gamma \rightarrow Q \rightarrow 1$ be a group extension which is associated with a holomorphic Seifert fibering $T_{\mathbb{C}}^{1} \rightarrow \mathcal{L} / \Gamma \rightarrow \mathbb{H}^{n} / Q$. As $\mathcal{L} / \mathbb{Z}^{2}$ is biholomorphic to the product $T_{\mathbb{C}}^{1} \times \mathbb{H}^{n}$, the holomorphic action of $Q$ can be described as

$$
\alpha(t, w)=(\phi(\alpha)(t) \cdot \chi(\alpha)(\alpha w), \alpha w)
$$

where $\chi(\alpha): \mathbb{H}^{n}=\mathbb{C}^{2 n} \rightarrow T_{\mathbb{C}}^{1}, \alpha \in Q$, is a holomorphic map. When $\mathcal{L} / \Gamma$ is described as $T_{\mathbb{C}}^{1} \times{ }_{Q} \mathbb{H}^{n}$, we introduce the following equivalence. 
Definition 6.3 Two such manifolds $\mathcal{L} / \Gamma_{1}, \mathcal{L} / \Gamma_{2}$ are equivalent if there is an equivariant fiber preserving holomorphic map $\mathrm{H}(t, w)=(t \cdot h(w), w)$ for a holomorphic map $h: \mathbb{H}^{n} \rightarrow T_{\mathbb{C}}^{1}$ with the following commutative diagram:

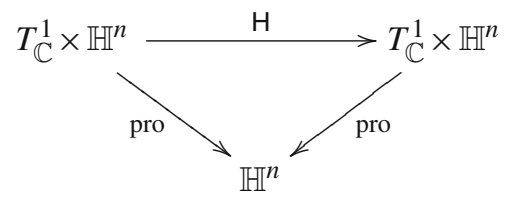

Let hol $\left(\mathbb{H}^{n}, \mathbb{C}\right)$ be the set of all holomorphic maps from $\mathbb{H}^{n}=\mathbb{C}^{2 n}$ to $\mathbb{C}$. It is a $Q$-module naturally. Similarly for $\operatorname{hol}\left(\mathbb{H}^{n}, T_{\mathbb{C}}^{1}\right)$ and $\mathbb{Z}^{2}$. Then a short exact sequence $1 \rightarrow \mathbb{Z}^{2} \stackrel{i}{\rightarrow} \operatorname{hol}\left(\mathbb{H}^{n}, \mathbb{C}\right) \stackrel{j}{\rightarrow} \operatorname{hol}\left(\mathbb{H}^{n}, T_{\mathbb{C}}^{1}\right) \rightarrow 1$ induces a long cohomology exact sequence [15]

$$
\longrightarrow H^{1}\left(Q ; \mathbb{Z}^{2}\right) \stackrel{i}{\longrightarrow} H^{1}\left(Q ; \operatorname{hol}\left(\mathbb{H}^{n}, \mathbb{C}\right)\right) \stackrel{j}{\longrightarrow} H^{1}\left(Q ; \operatorname{hol}\left(\mathbb{H}^{n}, T_{\mathbb{C}}^{1}\right)\right)
$$

$$
\longrightarrow H^{2}\left(Q ; \mathbb{Z}^{2}\right)
$$

The above group extension determines a 2-cocycle $[f] \in H^{2}\left(Q ; \mathbb{Z}^{2}\right)$. Consider the pre-image $\delta^{-1}([f]) \leq H^{1}\left(Q ; \operatorname{hol}\left(\mathbb{H}^{n}, T_{\mathbb{C}}^{1}\right)\right)$ which is thought of as a Jacobian variety. Then we have shown

Theorem 6.4 ([9]) The $Q$-module $\delta^{-1}([f])$ is the space of all equivalence classes (in the sense of Definition 6.3) representing $\mathcal{L} / \Gamma$.

- Each element of $\delta^{-1}([f])$ gives a complex contact Iwasawa infranilmanifold $\left(\mathcal{L} / \Gamma, J^{\prime}\right)$ which supports a holomorphic Seifert fibering $T_{\mathbb{C}}^{1} \rightarrow \mathcal{L} / \Gamma \longrightarrow \mathbb{H}^{n} / Q$.

- $\left(\mathcal{L} / \Gamma, J^{\prime}\right)$ is a deformation of $(\mathcal{L} / \Gamma, J)$ as a holomorphic $T_{\mathbb{C}}^{1}$-bundle.

We end this section with the proof of Theorem A.

\subsection{Proof of Theorem A}

As $M$ is a $(4 n+2)$-dimensional compact complex contact similarity manifold, by Definition 6.1 and Proposition 5.1, there is a developing pair

$$
(\rho, \mathrm{dev}):\left(\operatorname{Aut}_{\mathrm{cc}}(\tilde{M}), \widetilde{M}, J\right) \rightarrow\left(\operatorname{Sim}(\mathcal{L}), \mathcal{L}, J_{0}\right)
$$

Note that $\pi=\pi_{1}(M) \leq \operatorname{Aut}_{\mathrm{cc}}(\widetilde{M})$ and dev is a holomorphic immersion, i.e. $\operatorname{dev}_{*} J=$ $J_{0} \operatorname{dev}_{*}$. Put $\Gamma=\rho(\pi)$.

Assume (a). Taking a finite index subgroup we may assume $\Gamma$ is nilpotent. We prove this case by the following steps. 
Lemma 6.5 Suppose that some $\gamma=((a, w), \lambda \cdot A) \in \Gamma$ has a nontrivial summand $\lambda$, $\neq 1$, in $\mathbb{C}^{*} \leq \mathcal{L} \rtimes\left(\operatorname{Sp}(n) \cdot \mathbb{C}^{*}\right)=\operatorname{Sim}(\mathcal{L})$. Then $\Gamma \leq \operatorname{Sp}(n) \cdot \mathbb{C}^{*}$.

Proof As $\mathbb{C}^{*}=S^{1} \times \mathbb{R}^{+}$acts as multiplication on $\mathcal{L}$, it is noted that $\left[\mathcal{L}, \mathbb{R}^{+}\right]=\mathcal{L}($ and also $\left.\left[\mathcal{L}, S^{1}\right]=\mathcal{L}\right)$. So if $\Gamma$ has a nontrivial summand $x$ in $\mathcal{L}$, then $(1-\lambda \cdot A) x \in \Gamma_{1}=$ $[\Gamma, \Gamma]$ and so $(1-\lambda \cdot A)^{i} x \in \Gamma_{i}=\left[\Gamma_{i-1}, \Gamma\right]$ (possibly $\left.A=I\right)$. So $\Gamma_{i}=\left[\Gamma_{i-1}, \Gamma\right]$ cannot be trivial for any $i>0$. This is impossible because $\Gamma$ is nilpotent. Thus $\Gamma$ has no summand in $\mathcal{L}$ and hence

$$
\Gamma \leq \operatorname{Sp}(n) \cdot \mathbb{C}^{*} \leq \operatorname{Sim}(\mathcal{L}) .
$$

In particular, $\Gamma$ has the fixed point 0 at the origin $(0,0) \in \mathcal{L}$ (in this case $M$ is called radiant (compare [6])). We can assume that there exists an element $\gamma=(k, t) \in \Gamma$ such that

$$
k \in \operatorname{Sp}(n) \cdot S^{1}, \quad t \in \mathbb{R}^{+}, \quad t<1 .
$$

Lemma 6.6 The immersion $\operatorname{dev}$ misses $\{0\}$, i.e. $\{0\} \notin \operatorname{dev}(\widetilde{M})$.

Proof Assume $\{0\} \in \operatorname{dev}(\widetilde{M})$.

Case 1. If the complement $\mathcal{L}-\operatorname{dev}(\widetilde{M})$ is not empty, then it is a $\Gamma$-invariant closed subset which satisfies that $(\mathcal{L}-\operatorname{dev}(\widetilde{M})) \cap\{0\}=\emptyset$. Since both $\{0\}$ and $\mathcal{L}-\operatorname{dev}(\widetilde{M})$ are $\Gamma$-invariant, it follows

$$
\gamma^{i}(\mathcal{L}-\operatorname{dev}(\widetilde{M})) \cap\{0\}=\emptyset
$$

Choose a point $p=\left(z_{0}, z\right) \in \mathcal{L}-\operatorname{dev}(\widetilde{M})$. Then $\gamma^{i} p=\left(t^{4 i} z_{0}, t^{2 i} k^{2 i} z\right)$ by definition. It follows that $\lim _{i \rightarrow \infty} \gamma^{i} p=(0,0)=0 \in \mathcal{L}$ by (6). Since $\gamma^{i} p \in \mathcal{L}-\operatorname{dev}(\widetilde{M})$ which is a closed subset, it follows $0 \in \mathcal{L}-\operatorname{dev}(\tilde{M})$. This yields a contradiction. Hence Case 1 does not occur.

Case 2. Suppose $\mathcal{L}-\operatorname{dev}(\widetilde{M})=\emptyset$, i.e. dev is surjective. Recall that $M$ is viewed as an affinely flat manifold; $(\rho, \operatorname{dev}):(\pi, \widetilde{M}) \rightarrow\left(\Gamma, \mathbb{C}^{2 n+1}\right)$ where

$$
\Gamma=\left\{\left(\left[\begin{array}{l}
0 \\
0
\end{array}\right],\left(\begin{array}{c|c}
\lambda^{2} & 0 \\
\hline 0 & \lambda A
\end{array}\right)\right)\right\} \leq \operatorname{Aff}\left(\mathbb{C}^{2 n+1}\right) .
$$

If we note that $\Gamma$ is nilpotent, then it follows from [6, Theorem A] that $M$ is complete, i.e. the developing map dev: $\widetilde{M} \rightarrow \mathbb{C}^{2 n+1}$ is a diffeomorphism. Then $\Gamma \leq \operatorname{Sp}(n) \cdot \mathbb{C}^{*} \leq \operatorname{Sim}(\mathcal{L})$ is discrete and so a finite index subgroup of $\Gamma$ is an infinite cyclic subgroup. Then $\mathbb{C}^{2 n+1} / \Gamma$ cannot be compact and so Case 2 does not occur. As a consequence, combining Cases 1 and 2 shows that dev misses $\{0\}$.

Lemma 6.7 The manifold $M$ is biholomorphic to $S^{4 n+1} \times \mathbb{R}^{+} / \Gamma$ which is diffeomorphic to an infra-Hopf manifold $S^{4 n+1} / \mathbb{Z}_{p} \times S^{1}$.

Proof First note that the complement $\mathcal{L}-\{0\}$ admits an $\operatorname{Sp}(n) \cdot \mathbb{C}^{*}$-invariant Riemannian metric. In fact, $\mathbb{R}^{+} \leq \mathbb{C}^{*}$ acts on $\mathcal{L}-\{0\}$ as

$$
\lambda\left(z_{0}, z\right)=\left(\lambda^{2} z_{0}, \lambda z\right)
$$


so $\mathbb{R}^{+}$acts properly. Since $\operatorname{Sp}(n) \cdot S^{1}$ is compact, $\operatorname{Sp}(n) \cdot \mathbb{C}^{*}=\operatorname{Sp}(n) \cdot S^{1} \times \mathbb{R}^{+}$acts properly on $\mathcal{L}-\{0\}$. Then there exists an $\operatorname{Sp}(n) \cdot \mathbb{C}^{*}$-invariant Riemannian metric on $\mathcal{L}-\{0\}$ (see [13] for example). By Lemma 6.6, the developing image $\operatorname{dev}(\widetilde{M})$ misses 0 , i.e. $\operatorname{dev}(\widetilde{M}) \subset \mathcal{L}-\{0\}$. Taking a pullback metric by the developing immersion, $\operatorname{dev}: \widetilde{M} \rightarrow \mathcal{L}-\{0\}$ is a local isometry. Since this metric is $\Gamma$-invariant and dev is $\rho$-equivariant, the pullback metric on $\widetilde{M}$ is $\pi$-invariant which induces a Riemannian metric on the quotient $M$. As $M$ is compact, $M$ is complete with respect to this metric so is $\widetilde{M}$. Then $(\rho, \mathrm{dev}):(\pi, \widetilde{M}) \rightarrow(\Gamma, \mathcal{L}-\{0\})$ becomes an equivariant covering map. Since $\mathcal{L}-\{0\} \cong S^{4 n+1} \times \mathbb{R}^{+}$is simply connected, dev : $\widetilde{M} \rightarrow \mathcal{L}-\{0\}$ is a diffeomorphism. Taking the quotient, $M$ is holomorphically diffeomorphic to $\mathcal{L}-\{0\} / \Gamma$.

On the other hand, $\Gamma$ becomes discrete in $\operatorname{Sp}(n) \cdot S^{1} \times \mathbb{R}^{+}$:

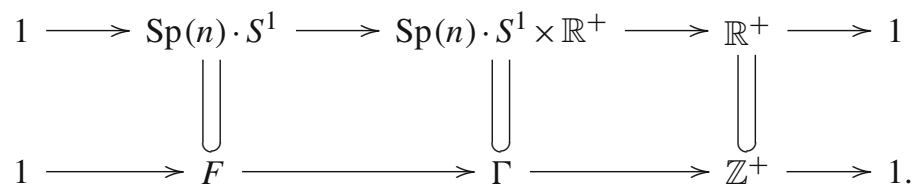

The image of $\Gamma$ to $\mathbb{R}^{+}$is nontrivial (and so an infinite cyclic subgroup $\mathbb{Z}^{+}$) because $\mathcal{L}-\{0\} / \Gamma$ is compact. Choosing an infinite cyclic subgroup $\mathbb{Z}^{+}$from $\Gamma$ which maps onto $\mathbb{Z}^{+} \leq \mathbb{R}^{+}$, it follows that $\Gamma=F \rtimes \mathbb{Z}^{+}$. Consider the exact sequences further,

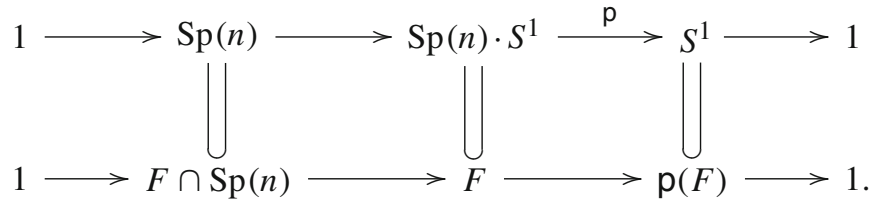

As $F \leq \Gamma$ acts freely on $\mathcal{L}-\{0\}$ in which $A \in \operatorname{Sp}(n)$ acts on $\mathcal{L}-\{0\}$ by $A\left(z_{0}, z\right)=$ $\left(z_{0}, A z\right)$ (cf. (7) of Case 2), it follows that $F \cap \operatorname{Sp}(n)=\{1\}$. In particular, $F \cong \mathrm{p}(F)$ is a finite cyclic subgroup of $S^{1}$. Put $F=\mathbb{Z}_{p}$, for some $p \in \mathbb{Z}$. As a consequence,

$$
\Gamma=\mathbb{Z}_{p} \times \mathbb{Z}^{+} \leq T^{2 n+1} \times \mathbb{R}^{+}
$$

which acts on $\mathcal{L}-\{0\}$ by

$$
\lambda\left(z_{0}, z_{1}, \ldots, z_{2 n}\right)=\left(\lambda^{2} z_{0}, \lambda z_{1}, \ldots, \lambda z_{2 n}\right) .
$$

As the $\mathbb{Z}_{p}$-action preserves the standard sphere $S^{4 n+1} \subset \mathcal{L}-\{0\}=S^{4 n+1} \times \mathbb{R}^{+}$, the $\Gamma$-action on $\mathcal{L}-\{0\}$ induces a properly discontinuous action of $\mathbb{Z}^{+}$on $S^{4 n+1} / \mathbb{Z}_{p} \times \mathbb{R}^{+}$ (here $S^{4 n+1} / \mathbb{Z}_{p}$ is the lens space $L(p, 2,1, \ldots, 1)$ ). Hence $\mathcal{L}-\{0\} / \Gamma$ is biholomorphic to an infra-Hopf complex contact manifold $S^{4 n+1} / \mathbb{Z}_{p} \times S^{1}$.

There is the remaining case to Lemma 6.5. 
Lemma 6.8 Suppose $\Gamma \leq \mathcal{L} \rtimes \operatorname{Sp}(n) \cdot S^{1} \leq \operatorname{Sim}(\mathcal{L})$, i.e. every $\gamma$ has no summand in $\mathbb{R}^{+}$. Then $M$ is biholomorphic to an infranilmanifold $\mathcal{L} / \Gamma$.

Proof Since $\mathcal{L} \rtimes \operatorname{Sp}(n) \cdot S^{1}$ acts properly on $\mathcal{L}$, there is an $\mathcal{L} \rtimes \operatorname{Sp}(n) \cdot S^{1}$-invariant Riemannian metric on $\mathcal{L}$. Taking the pullback metric, it follows similarly as above that $(\rho, \mathrm{dev}):(\pi, \widetilde{M}) \rightarrow\left(\mathcal{L} \rtimes \mathrm{Sp}(n) \cdot S^{1}, \mathcal{L}\right)$ becomes an equivariant isometry. Hence $M$ is holomorphically diffeomorphic to $\mathcal{L} / \Gamma$. Auslander-Bieberbach's theorem implies that $\mathcal{L} / \Gamma$ is finitely covered by a holomorphic nilmanifold $\mathcal{L} / \Delta$, where $\Delta=\Gamma \cap \mathcal{L}$ is a finite index normal subgroup of $\Gamma$. Hence $\mathcal{L} / \Gamma$ is a holomorphic infranilmanifold.

All together with Lemmas 6.5, 6.6, 6.7 and 6.8, this finishes the proof of Theorem A under the condition $(a)$.

Assume (b). Since $\operatorname{Sim}(\mathcal{L})=\operatorname{Sp}(n) \cdot S^{1} \times \mathbb{R}^{+}$is an amenable Lie group, any discrete subgroup is virtually polycyclic (see [16]). We may assume that $\Gamma$ is polycyclic. Consider the exact sequences

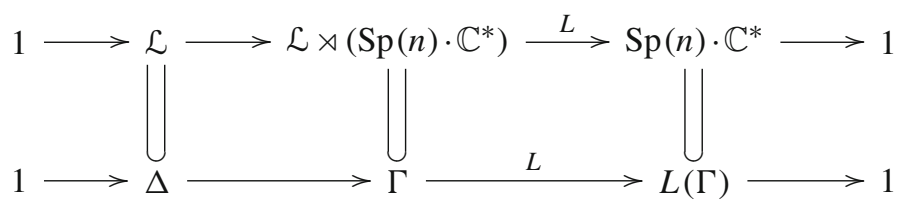

where we put $\Delta=\mathcal{L} \cap \Gamma$. As $L(\Gamma)$ is solvable, it follows that $L(\Gamma) \leq T^{n} \times S^{1} \times \mathbb{R}^{+}$. In particular, $[\Gamma, \Gamma] \leq \Delta$. We can assume that $\Delta$ is nontrivial. Otherwise, $[\Gamma, \Gamma]=\{1\}$, $\Gamma$ is abelian. So the condition Theorem A(a) is satisfied. Then some finite cover of $M$ is either a complex contact nilmanifold $\mathcal{L} / \Gamma$ or a complex contact Hopf manifold $S^{4 n+1} \times S^{1}$. As $\Gamma$ is abelian, some finite cover of $M$ must be a complex contact Hopf manifold $S^{4 n+1} \times S^{1}$.

Suppose that there is an element $\gamma \in \Gamma$ such that $L(\gamma)$ has a nontrivial summand $\lambda$ in $\mathbb{R}^{+}$. We may assume that $\lambda<1$. Put $\gamma=((a, w), \lambda A)$ and $n=(b, z) \in \Delta$. We can check that

$$
\begin{aligned}
\gamma^{i} n \gamma^{-i} & =\left(\lambda^{2 i} b+2 \sum_{k=1}^{i} \lambda^{2 i-k}\left({ }^{t} w J_{n} A^{k} z\right), \lambda^{i} A^{i} z\right) \\
& =\left(\lambda^{i}\left(\lambda^{i} b+2 \sum_{k=1}^{i} \lambda^{i-k}\left({ }^{t} w J_{n} A^{k} z\right)\right), \lambda^{i} A^{i} z\right) .
\end{aligned}
$$

As $\gamma^{i} n \gamma^{-i} \in \Delta$, it follows

$$
\lim _{i \rightarrow \infty} \gamma^{i} n \gamma^{-i}=(0,0)=1 \in \Delta
$$

which contradicts that $\Delta$ is discrete. Hence $L(\Gamma) \leq T^{n} \times S^{1}$ and so $\Gamma \leq$ $\mathcal{L} \rtimes T^{n} \times S^{1} \leq \mathcal{L} \rtimes \mathrm{Sp}(n) \cdot S^{1}$. As in the proof of Lemma $6.8, M$ is biholomorphic to an infranilmanifold $\mathcal{L} / \Gamma$. 
This proves Theorem A with conditions (a) or (b) respectively. We proved this theorem in [10] when $S^{1} \leq \operatorname{Aut}_{\mathrm{cc}}(M)$ acts on $M$ without fixed points.

\section{Compactification of $\mathcal{L}$ and developing maps}

\section{$7.1(4 n+2)$-sphere $\mathcal{L} \cup\{\infty\}$}

Let $\left(\operatorname{PSp}(n+1,1), \mathbb{H}_{\mathbb{H}}^{n+1}\right)$ be the homogeneous model of the quaternionic hyperbolic geometry. There is a compactification $\overline{\mathbb{H}}_{\mathbb{H}}^{n+1}$ of $\mathbb{H}_{\mathbb{H}}^{n+1}$ whose boundary $\partial \mathbb{H}_{\mathbb{H}}^{n+1}$ is diffeomorphic to the $(4 n+3)$-sphere $S^{4 n+3}$. The isometric group $\operatorname{PSp}(n+1,1)$ extends to a projective action on $S^{4 n+3}$ whose stabilizer at the point at infinity $\infty \in S^{4 n+3}$ is isomorphic to the maximal amenable Lie group $\mathcal{M} \rtimes \operatorname{Sp}(n, 1) \cdot \operatorname{Sp}(1) \times \mathbb{R}^{+}$, where $\mathcal{M}$ is the quaternionic Heisenberg Lie group whose center is $\mathbb{R}^{3}$. There is the parabolic decomposition $S^{4 n+3}=\mathcal{M} \cup\{\infty\}$. As $\mathbb{R} \leq \mathcal{M}$ acts as isometries of $\mathbb{H}_{\mathbb{H}}^{n+1}$, there is the principal bundle $\mathbb{R} \rightarrow \mathbb{H}_{\mathbb{H}}^{n+1} \stackrel{p}{\rightarrow} B$, where $B=\mathbb{H}_{\mathbb{H}}^{n+1} / \mathbb{R}$. Then the projection $p$ naturally extends to a map $\bar{p}$ of $\overline{\mathbb{H}}_{\mathbb{H}}^{n+1}$ in such a way that

$$
\bar{p}: \mathbb{H}_{\mathbb{H}}^{n+1} \cup(\mathcal{M} \cup\{\infty\}) \rightarrow B \cup(\mathcal{L} \cup\{\infty\})
$$

where $\mathbb{R} \rightarrow \mathcal{M} \stackrel{p}{\rightarrow} \mathcal{L}$ is the exact sequence as before. Then note that $\bar{B}=B \cup(\mathcal{L} \cup\{\infty\})$ is homeomorphic to a $(4 n+3)$-ball such that the boundary $\partial B=\mathcal{L} \cup\{\infty\}$ is a sphere $S^{4 n+2}$. Thus we have an equivariant projection

$$
\mathbb{R} \rightarrow\left(\mathcal{M} \rtimes \operatorname{Sp}(n) \cdot \mathbb{C}^{*}, \overline{\mathbb{H}}_{\mathbb{H}}^{n+1}\right) \stackrel{\bar{p}}{\longrightarrow}\left(\mathcal{L} \rtimes \operatorname{Sp}(n) \cdot \mathbb{C}^{*}, \bar{B}\right)
$$

\subsection{A proper action on a domain of $\bar{B}$}

Suppose that a group $\Gamma \leq \mathcal{L} \rtimes \operatorname{Sp}(n) \cdot \mathbb{C}^{*}$ acts on a domain $\Omega$ of $\mathcal{L}$. We note that the action of $\Gamma$ naturally extends to an action on $\partial B=\mathcal{L} \cup\{\infty\}$ such that $\Gamma$ leaves $\infty$ fixed. Define

$$
\partial \Omega=\partial B-\Omega(=(\mathcal{L}-\Omega) \cup\{\infty\}) .
$$

Then $\partial \Omega$ is a closed subset in $\partial B$. Let $\bar{\Gamma} \leq \mathcal{L} \rtimes \operatorname{Sp}(n, 1) \cdot \mathbb{C}^{*}$ be the closure of $\Gamma$. As $\partial \Omega$ is closed and $\Omega$ is $\Gamma$-invariant, note that $\bar{\Gamma}$ leaves $\partial \Omega$ invariant.

Proposition 7.1 Suppose that $\mathcal{L}-\Omega \neq \emptyset$. Then either one of the following holds: (i) $\bar{\Gamma} \leq \mathcal{L} \rtimes \operatorname{Sp}(n) \cdot S^{1}$ or (ii) there exists a $\bar{\Gamma}$-invariant simply connected domain $Y \subset \mathcal{L}$ containing $\Omega$.

Proof Let $1 \rightarrow \mathbb{R} \rightarrow \mathcal{M} \rtimes \operatorname{Sp}(n) \cdot \mathbb{C}^{*} \stackrel{\nu}{\rightarrow} \mathcal{L} \rtimes \operatorname{Sp}(n) \cdot \mathbb{C}^{*} \rightarrow 1$ be the exact sequence. Put $G=v^{-1}(\bar{\Gamma})$ and $\Lambda=\bar{p}^{-1}(\partial \Omega)$. Then $\Lambda$ is a $G$-invariant closed subset in $S^{4 n+3}$. 
Since $\mathcal{L}-\Omega \neq \emptyset$, note that $\Lambda \varsubsetneqq S^{4 n+3}$. Let $L(G)$ be the limit set for $G$ (compare [2]). As $\Lambda$ contains more than one point, it follows $L(G) \subset \Lambda$. Consider the exact sequence

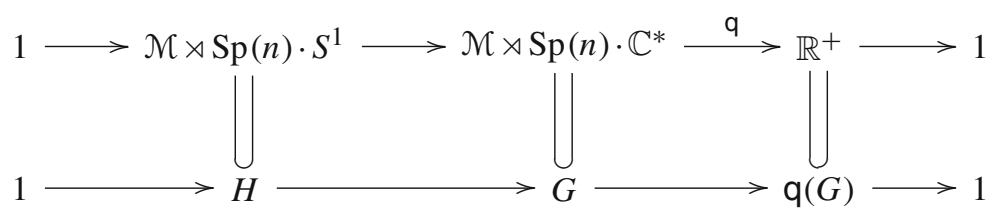

where $H=G \cap\left(\mathcal{M} \rtimes \operatorname{Sp}(n) \cdot S^{1}\right)$.

Suppose that $\mathrm{q}(G) \neq\{1\}$ otherwise (i) holds. Let $G^{0}$ be the identity component of $G$.

Case 1. Suppose that $\mathrm{q}\left(G^{0}\right)=\mathbb{R}^{+}$. Then $G=H \rtimes \mathbb{R}^{+}$. As $G^{0}=H^{0} \rtimes \mathbb{R}^{+}$, it follows (cf. [11])

$$
L(G)=L\left(H^{0} \rtimes \mathbb{R}^{+}\right) .
$$

Case 2. Suppose that $G^{0} \leq \mathcal{M} \rtimes \operatorname{Sp}(n, 1) \cdot S^{1}$. The above exact sequence induces a group extension

$$
1 \rightarrow H / G^{0} \rightarrow G / G^{0} \stackrel{\mathrm{q}}{\rightarrow} \mathrm{q}(G) \rightarrow 1 .
$$

As $\mathrm{q}(G) \leq \mathbb{R}^{+}$and $H / G^{0}$ is discrete, noting that $\mathbb{R}^{+}$acts on $\mathcal{M}$ as multiplication: $\lambda(a, z)=\left(\lambda^{2} a, \lambda z\right), \lambda \in \mathbb{R}^{+},(a, z) \in \mathcal{M}$, it follows $H / G^{0}=\{1\}$, or $H=G^{0}$. Then $\mathrm{q}(G)$ is discrete in $\mathbb{R}^{+}$and so $\mathrm{q}(G) \cong \mathbb{Z}^{+}$. It follows $G=H^{0} \rtimes \mathbb{Z}^{+}$and so

$$
L(G)=L\left(H^{0} \rtimes \mathbb{Z}^{+}\right) .
$$

Let $\left(G, \overline{\mathbb{H}}_{\mathbb{H}}^{n+1}\right)$ be the action as above, where $G \leq \mathcal{M} \rtimes \operatorname{Sp}(n, 1) \cdot \mathbb{C}^{*}$. The totally geodesic subspace of $\mathbb{H}_{\mathbb{H}}^{n+1}$ is $\mathbb{H}_{\mathbb{K}}^{m}$ for $\mathbb{K}=\mathbb{R}, \mathbb{C}, \mathbb{H}, 1 \leq m \leq n+1$. If we note that $\mathbb{R} \leq H^{0} \leq \mathcal{M} \rtimes \operatorname{Sp}(n, 1) \cdot S^{1}$, then $H^{0} \rtimes \mathbb{R}^{+}$is viewed as a warped product which acts transitively in $\mathbb{H}_{\mathbb{K}}^{m}$. Similarly, $H^{0} \rtimes \mathbb{Z}^{+}$acts in $\mathbb{H}_{\mathbb{K}}^{m}$ for which the orbit (at a point in $\mathbb{H}_{\mathbb{K}}^{m}$ ) approaches to the boundary $\partial \mathbb{H}_{\mathbb{K}}^{m}$. By the definition of the limit set (cf. [2]), both Cases 1 and 2 imply that

$$
L(G)=\partial \mathbb{H}_{\mathbb{K}}^{m}
$$

for $\mathbb{K}=\mathbb{R}, \mathbb{C}, \mathbb{H}$ and $1 \leq m \leq n+1,1 \leq m \leq n+1,1 \leq m \leq n$ respectively. For the last case, $m=n+1$ is excluded because $\Lambda \varsubsetneqq S^{4 n+3}$ (as $\mathbb{R} \leq H^{0}$, note that $\partial\left(\mathbb{R} \rtimes \mathbb{R}^{+} \cdot p\right)=\partial\left(\mathbb{R} \rtimes \mathbb{Z}^{+} \cdot p\right)=\partial \mathbb{H}_{\mathbb{R}}^{2}=S^{1}$ for $p \in \mathbb{H}_{\mathbb{H}}^{n+1}$. In particular, $\{\infty\} \in$ $\left.S^{1} \subset L(G)\right)$. Then the complement $S^{4 n+3}-L(G)$ is homeomorphic to $\mathbb{H}_{\mathbb{K}}^{m} \times S^{\ell}$, where $\ell=4 n+3-m|\mathbb{K}|$ for $|\mathbb{K}|=1,2$ or 4 . Since $\ell \geq 3 n+2,2 n+1,3$ respectively for $|\mathbb{K}|=1,2$ or $4, S^{4 n+3}-L(G)$ is a simply connected $G$-invariant submanifold.

Moreover, as $S^{4 n+3}-L(G)=S^{4 n+3}-S^{m|\mathbb{K}|-1}$, if $\operatorname{Aut}\left(S^{4 n+3}-L(G)\right)$ is a subgroup of $\operatorname{PSp}(n+1,1)$ leaving $S^{4 n+3}-L(G)$ invariant, then it acts properly on $S^{4 n+3}-L(G)$ (compare [8,14]). This follows also from the result of Frances [4] which says that if 
a domain $D$ of $S^{4 n+3}$ is not $S^{4 n+3}, \mathcal{M}$, then Aut $(D)$ acts properly on $\left.D\right)$. Since $G$ is a closed subgroup of Aut $\left(S^{4 n+3}-L(G)\right), G$ acts properly on $S^{4 n+3}-L(G)$.

As $\infty \in L(G)$, note that $S^{4 n+3}-L(G)=(\mathcal{M} \cup \infty)-L(G) \subset \mathcal{M}$. Since $\mathbb{R}$ $(\leq G)$ acts properly and freely on $\mathcal{M}$, we have a simply connected manifold $Y=$ $S^{4 n+3}-L(G) / \mathbb{R}$ which is a submanifold of $\mathcal{L}$. There induces an equivariant principal bundle:

$$
\mathbb{R} \rightarrow\left(G, S^{4 n+3}-L(G)\right) \stackrel{(\nu, \bar{p})}{\longrightarrow}(\bar{\Gamma}, Y) .
$$

Then it is easy to see that $\bar{\Gamma}$ acts properly on $Y$. There is a $\bar{\Gamma}$-invariant Riemannian metric on $Y$ (compare [13]). Recall that $L(G) \subset \Lambda$, then

$$
\Omega \subset S^{4 n+2}-\partial \Omega=\bar{p}\left(S^{4 n+3}-\Lambda\right) \subset \bar{p}\left(S^{4 n+3}-L(G)\right)=Y .
$$

We obtain $(\Gamma, \Omega) \subset(\bar{\Gamma}, Y)$.

Theorem 7.2 If the developing map of a compact $(4 n+2)$-dimensional complex contact similarity manifold $M$ is not surjective onto $\mathcal{L}$, then $\mathrm{dev}$ is a biholomorphim of $\widetilde{M}$ onto $\mathcal{L}-\{0\}$. In particular, $M$ is a complex contact infra-Hopf manifold.

Proof We have a developing pair dev: $(\pi, \widetilde{M}) \longrightarrow(\Gamma, \mathcal{L})$. Suppose that dev is not surjective onto $\mathcal{L}$. Let $\Omega=\operatorname{dev}(\tilde{M})$. Then $\mathcal{L}-\Omega=\partial \Omega \neq \emptyset$. By Proposition 7.1, there is a $\bar{\Gamma}$-invariant Riemannian metric on $Y$ such that the pull back metric by dev induces a local isometry dev $:(\pi, \widetilde{M}) \longrightarrow(\bar{\Gamma}, Y)$. As $M$ is compact and $Y$ is simply connected, dev: $\widetilde{M} \longrightarrow Y$ is an isometry. Hence $(\Gamma, \Omega)=(\bar{\Gamma}, Y)$. In particular, $\Gamma$ is discrete. Then the result follows from Theorem A.

\section{References}

1. Alekseevsky, D., Kamishima, Y.: Pseudo-conformal quaternionic $C R$ structure on $(4 n+3)$-dimensional manifolds. Ann. Mat. Pura Appl. 187(3), 487-529 (2008)

2. Chen, S.S., Greenberg, L.: Hyperbolic spaces. In: Ahlfors, L.V., Kra, I., Maskit, B., Nirenberg, L. (eds.) Contributions to Analysis, pp. 49-87. Academic Press, New York (1974)

3. Foreman, B.: Boothby-Wang fibrations on complex contact manifolds. Differential Geom. Appl. 13(2), 179-196 (2000)

4. Frances, C.: Sur le groupe d'automorphismes des géométries paraboliques de rang 1. Ann. Sci. École Norm. Sup. 40(5), 741-764 (2007)

5. Fried, D.: Closed similarity manifolds. Comment. Math. Helv. 55(4), 576-582 (1980)

6. Fried, D., Goldman, W., Hirsch, M.W.: Affine manifolds with nilpotent holonomy. Comment. Math. Helv. 56(4), 487-523 (1981)

7. Ishihara, S., Konishi, M.: Real contact 3-structure and complex contact structure. Southeast Asian Bull. Math. 3(2), 151-161 (1979)

8. Kamishima, Y.: Geometric flows on compact manifolds and global rigidity. Topology 35(2), 439-450 (1996)

9. Kamishima, Y., Nakayama, M.: On the holomorphic torus-Bott tower of aspherical manifolds. Proc. Steklov Inst. Math. 286(1), 253-267 (2014)

10. Kamishima, Y., Tanaka, A.: On complex contact similarity manifolds. J. Math. Res. 5(4), 1-10 (2013)

11. Kamishima, Y., Udono, T.: Three-dimensional Lie group actions on compact $(4 n+3)$-dimensional geometric manifolds. Differential Geom. Appl. 21(1), 1-26 (2004)

12. Kobayashi, S.: Remarks on complex contact manifolds. Proc. Amer. Math. Soc. 10, 164-167 (1959)

13. Koszul, J.-L.: Lectures on Groups of Transformations. Tata Institute of Fundamental Research Lectures on Mathematics, vol. 32. Tata Institute of Fundamental Research, Bombay (1965) 
14. Kulkarni, R.S.: On the principle of uniformization. J. Differential Geom. 13(1), 109-138 (1978)

15. Lee, K.B., Raymond, F.: Seifert fiberings. Mathematical Surveys and Monographs, vol. 166. American Mathematical Society, Providence (2010)

16. Milnor, J.: On fundamental groups of complete affinely flat manifolds. Adv. Math. 25(2), 178-187 (1977)

17. Miner, R.R.: Spherical CR manifolds with amenable holonomy. Internat. J. Math. 1(4), 479-501 (1990)

18. Wolf, J.A.: Complex homogeneous contact manifolds and quaternionic symmetric spaces. J. Math. Mech. 14(6), 1033-1047 (1965) 